\title{
Refinement of an estimate for the Hurwitz zeta function in a neighbourhood of the line $\sigma=1$
}

\author{
by \\ MieczysŁaW Kulas (Poznań)
}

The well-known estimate of the order of the Hurwitz zeta function

$$
\zeta(s, \alpha)-\alpha^{-s} \ll t^{c(1-\sigma)^{3 / 2}} \log ^{2 / 3} t
$$

is proved with the constant $c=18.4974$ for $1 / 2 \leq \sigma \leq 1, t \geq t_{0}>0$.

The improvement of the constant $c$ is a consequence of some technical modifications in the method of estimating exponential sums sketched by Heath-Brown ([11], p. 136).

I. Introduction. In 1967 H. E. Richert [9] proved for the Hurwitz zeta function (defined in the half plane $\operatorname{Re}(s)>1$ by $\zeta(s, \alpha)=\sum_{n=0}^{\infty}(n+\alpha)^{-s}$, $0<\alpha \leq 1)$ that

$$
\left|\zeta(s, \alpha)-\alpha^{-s}\right| \leq c_{0} t^{c(1-\sigma)^{3 / 2}} \log ^{2 / 3} t
$$

for $1 / 2 \leq \sigma \leq 1, t \geq 2$, where $c_{0}$ is an absolute positive constant and $c=100$. This leads to the same bound for the Riemann zeta function,

$$
|\zeta(\sigma+i t)| \leq c_{1} t^{100(1-\sigma)^{3 / 2}} \log ^{2 / 3} t
$$

for $1 / 2 \leq \sigma \leq 1, t \geq 2$ and a positive constant $c_{1}$.

More generally, one can deduce from (1) that if $L(s, \chi)$ denotes the Dirichlet $L$-function associated with the Dirichlet character $\chi(\bmod k)$, $k \geq 1$, then

$$
|L(\sigma+i t, \chi)| \leq c_{2} k^{1-\sigma} t^{100(1-\sigma)^{3 / 2}} \log ^{2 / 3} t+k^{1-\sigma} \log k
$$

for the same range as (2) and a positive constant $c_{2}$.

The bounds of the type (2) or (3) have existed for a long time in the literature and have various applications (zero-free regions, a problem of Dirichlet divisors in number fields, the order of the Dedekind zeta function of a quadratic field, and so on).

1991 Mathematics Subject Classification: Primary 11M35. 
Several authors have reduced the constant $c=100$ in (2) or (3). For example, $c=86$ [2], $c=39$ [12] (also compare [4], Chapter 6 with $c=122$, $\left.c=2^{15}[10]\right)$.

In 1988 using Tyrina's version (see [13]) of Vinogradov's mean value theorem, Panteleeva [6] proved that $c=21$ in (3) but it seems that this result is incorrect (since Tyrina's result has a factor $n^{4 k^{2}}$ in the "constant" and it is not clear how to make it an absolute constant). Note that in [7] (1994) Panteleeva postulated $c=21.57$.

Heath-Brown ([11], p. 135) pointed out that "the best result up-to-date appears to be one in which 100 is replaced by 18.8" (Heath-Brown, unpublished).

In this paper we will show that Richert's result (1) can be sharpened for a given range of $\sigma$ and sufficiently large $t>0$. We shall prove the following

TheOREM. If $s=\sigma+i t$ and $0<\alpha \leq 1$, then there exists an absolute positive constant $c_{0}$ such that

$$
\left|\zeta(s, \alpha)-\alpha^{-s}\right| \leq c_{0} t^{c(1-\sigma)^{3 / 2}} \log ^{2 / 3} t
$$

for $1 / 2 \leq \sigma \leq 1, t \geq t_{0}>0$ and $c=18.4974$.

The improvement of the constant $c$ is a consequence of some technical modifications in the method of estimating exponential sums sketched by Heath-Brown ([11], p. 136).

Perhaps, the latest developments in the theory of I. M. Vinogradov's mean value theorem (due to T. Wooley and others) could be used to obtain an even better value of $c$. Of course the up-to-date constant $c=18.4974$ is still large, particularly in view of the fact that according to the Riemann hypothesis it should tend to zero.

II. Lemmas. In the proof of the Theorem we use some lemmas, presented below for convenience. We suppose that $s=\sigma+i t$ and $0<\alpha \leq 1$. All constants occurring in the Vinogradov symbol $\ll$ are absolute.

Lemma 1 (compare [9], p. 101). For $0<\sigma \leq 1, t \geq t_{1}>0$,

$$
\sum_{n \leq \exp \left(\log ^{2 / 3} t\right)}(n+\alpha)^{-s} \ll t^{(1-\sigma)^{3 / 2}} \log ^{2 / 3} t .
$$

Lemma 2 ([5], p. 124). Let $\sigma>0, t \geq t_{2}>0, M, N \in \mathbb{N}, N<M \leq 2 N$, $\exp \left(\log ^{2 / 3} t\right)<N \leq t^{1 / 1000}$. Then there exist positive constants $\gamma, \delta$ such that

$$
\left|\sum_{N<n \leq M}(n+\alpha)^{-s}\right| \leq \gamma N^{1-\sigma-\delta\left(\frac{\log N}{\log t}\right)^{2}}
$$

where $\gamma=2.003$ and $\delta=(2309.525)^{-1}$. 
This lemma plays the main role in the proof of our Theorem. The latest version of Vinogradov's mean value theorem joined with some technical modifications in the method of estimating exponential sums (Heath-Brown $[11]$, p. 136) enables us to get a very good value of the constant $\delta$. Numerical calculations show that we can get a very small improvement of $\delta$ if we decrease the exponent $1 / 1000$ of $t$, so in this light our choice of the range of $N$ seems to be optimal.

Corollary 1. For $0<\sigma \leq 1$ and $t \geq t_{3}>0$,

$$
S=\sum_{\exp \left(\log ^{2 / 3} t\right)<n \leq t^{1 / 1000}}(n+\alpha)^{-s} \ll t^{B(1-\sigma)^{3 / 2}} \log ^{2 / 3} t,
$$

where $B=18.4974$.

Proof. Let

$$
Q=\left[\exp \left(\log ^{2 / 3} t\right)\right]+1
$$

We see that

$$
S=(Q+\alpha)^{-s}+\sum_{Q<n \leq t^{1 / 1000}}(n+\alpha)^{-s}
$$

Let $r$ be the largest integer such that $Q \cdot 2^{r}<T^{1 / 1000}$. Then

(2)

$$
\begin{aligned}
& \sum_{Q<n \leq t^{1 / 1000}}(n+\alpha)^{-s} \\
& =\sum_{m=0}^{r-1} \sum_{Q \cdot 2^{m}<n \leq Q \cdot 2^{m+1}}(n+\alpha)^{-s}+\sum_{Q \cdot 2^{r}<n \leq t^{1 / 1000}}(n+\alpha)^{-s} .
\end{aligned}
$$

From Lemma 2 we get

(3)

$$
\begin{aligned}
\sum_{Q<n \leq t^{1 / 1000}} & (n+\alpha)^{-s} \\
& \ll \sum_{m=0}^{r}\left(Q \cdot 2^{m}\right)^{1-\sigma-\delta\left(\frac{\log Q \cdot 2^{m}}{\log t}\right)^{2}} \\
& =\sum_{m=0}^{r} \exp \left\{(1-\sigma) \log \left(Q \cdot 2^{m}\right)-\delta \frac{\log ^{3} Q \cdot 2^{m}}{\log ^{2} t}\right\}=S_{1} .
\end{aligned}
$$

If $\delta=(2309.525)^{-1}=\delta_{1}+\delta_{2}, \delta_{1}, \delta_{2}>0$, then

$$
\begin{aligned}
S_{1}= & \sum_{m=0}^{r} \exp \left\{(1-\sigma) \log \left(Q \cdot 2^{m}\right)-\delta_{1} \frac{\log ^{3} Q \cdot 2^{m}}{\log ^{2} t}\right\} \\
& \times \exp \left\{-\delta_{2} \frac{\log ^{3} Q \cdot 2^{m}}{\log ^{2} t}\right\} .
\end{aligned}
$$


Considering the function $f(x)=(1-\sigma) x-\delta_{1} x^{3} / \log ^{2} t, x>0$, we shall see that $f$ has a maximum at the point $x_{0}=\sqrt{\frac{1-\sigma}{3 \delta_{1}}} \log t$ and $f\left(x_{0}\right)=$ $\frac{2}{3}\left(\sqrt{3 \delta_{1}}\right)^{-1}(1-\sigma)^{3 / 2} \log t$. This implies that

$$
S_{1} \leq\left(\sum_{m=0}^{r} \exp \left\{-\delta_{2} \frac{\log ^{3} Q \cdot 2^{m}}{\log ^{2} t}\right\}\right) t^{(2 / 3)\left(\sqrt{3 \delta_{1}}\right)^{-1}(1-\sigma)^{3 / 2}} .
$$

Now

$$
\begin{aligned}
\sum_{m=0}^{r} \exp \left\{-\delta_{2} \frac{\log ^{3} Q \cdot 2^{m}}{\log ^{2} t}\right\} & \\
\leq & \exp \left\{-\delta_{2} \frac{\log ^{3} Q}{\log ^{2} t}\right\}+\int_{0}^{r} \exp \left\{-\delta_{2} \frac{\log ^{3} Q \cdot 2^{x}}{\log ^{2} t}\right\} d x \\
& <e^{-\delta_{2}}+\int_{0}^{\infty} \exp \left\{-\delta_{2} \frac{\log ^{3} Q \cdot 2^{x}}{\log ^{2} t}\right\} d x \\
& \leq e^{-\delta_{2}}+1.5(\log 2)^{-1}\left(\frac{\log ^{2} t}{\delta_{2}}\right)^{1 / 3} .
\end{aligned}
$$

Choose the parameters $\delta_{1}, \delta_{2}$ in the way that $\delta_{1}=(2309.526)^{-1}$. This gives

$$
\frac{2}{3}\left(\sqrt{3 \delta_{1}}\right)^{-1}=18.497351 \ldots<18.4974=B
$$

and $\delta_{2}=\delta-\delta_{1}>10^{-3} \cdot(2309.526)^{-2}$.

From (4)-(7) we see that

$$
S_{1} \ll t^{B(1-\sigma)^{3 / 2}} \log ^{2 / 3} t
$$

and from (1)-(3) and (8) we have

$$
S \ll Q^{-\sigma}+t^{B(1-\sigma)^{3 / 2}} \log ^{2 / 3} t \ll t^{B(1-\sigma)^{3 / 2}} \log ^{2 / 3} t .
$$

Lemma 3 ([9], Hilfssatz 4). For $1-1 / 2^{r+1} \leq \sigma, 4 \leq r \leq \log \log t$ and $t \geq t_{4}>0$

$$
\sum_{t^{1 /(r-1)}<n \leq t^{1 / 2}}(n+\alpha)^{-s}=O(1) .
$$

From Lemma 3 we get immediately:

Corollary 2. For $1-2^{-1002} \leq \sigma$ and $t \geq t_{5}>0$,

$$
\sum_{t^{1 / 1000}<n \leq t^{1 / 2}}(n+\alpha)^{-s}=O(1) .
$$

According to Vinogradov's theorem in the form given in [14] (Th. 1b, p. 114, compare an example after that theorem) one can easily achieve the following: 
Lemma 4. Let $\sigma>0, t \geq t_{6}>0, k, P_{0}, P \in \mathbb{N}, k \geq 11,0<P_{0} \leq P$ and $t^{1 / k} \leq P \leq t^{1 /(k-1)}$. Then

$$
\sum_{P \leq n \leq P+P_{0}-1}(n+\alpha)^{-s} \ll(8 k)^{(k \log 120 k) / 2} P^{1-\sigma-\varrho},
$$

where $\varrho=\left(3 k^{2} \log 120 k\right)^{-1}$.

As an application of this lemma one can obtain

Corollary 3. For $0<\sigma<1-2^{-1002}$ and $t \geq t_{7}>0$,

$$
\sum_{t^{1 / 1000}<n \leq t^{1 / 10}}(n+\alpha)^{-s} \ll t^{18.1(1-\sigma)^{3 / 2}} \log ^{2 / 3} t .
$$

Proof. Consider a sum $\sum_{t^{1 / k}<n \leq t^{1 /(k-1)}}(n+\alpha)^{-s}, 11 \leq k \leq 1000$. If $Q=\left[t^{1 / k}\right]+1$ and $r$ denotes the largest integer such that $Q \cdot 2^{r}<$ $t^{1 /(k-1)}$, then

$$
\begin{aligned}
& \sum_{t^{1 / k}<n \leq t^{1 /(k-1)}}(n+\alpha)^{-s} \\
& \quad=\sum_{m=0}^{r-1} \sum_{Q \cdot 2^{m} \leq n \leq Q \cdot 2^{m+1}-1}(n+\alpha)^{-s}+\sum_{Q \cdot 2^{r} \leq n \leq t^{1 /(k-1)}}(n+\alpha)^{-s} .
\end{aligned}
$$

With the help of Lemma 4 we see that

$$
\sum_{t^{1 / k}<n \leq t^{1 /(k-1)}}(n+\alpha)^{-s} \ll \sum_{m=0}^{r}\left(Q \cdot 2^{m}\right)^{1-\sigma-\varrho}, \quad \varrho=\left(3 k^{2} \log 120 k\right)^{-1},
$$

because the dependence on $k$ can be incorporated in the order constant $(k \leq 1000)$. then

Clearly $2^{-1002}-\varrho<1-\sigma-\varrho<1-\varrho$ and $r \ll \log t$. If $1-\sigma-\varrho \leq 0$

$$
\begin{aligned}
\sum_{t^{1 / k}<n \leq t^{1 /(k-1)}}(n+\alpha)^{-s} & \ll \log t \ll t^{18(1-\sigma)^{3 / 2}} \log t \\
& \ll t^{18.1(1-\sigma)^{3 / 2}} \log ^{2 / 3} t
\end{aligned}
$$

Otherwise

$$
\begin{aligned}
\sum_{t^{1 / k}<n \leq t^{1 /(k-1)}}(n+\alpha)^{-s} & \ll t^{(1 /(k-1))(1-\sigma-\varrho)} \log t \\
& =t^{18(1-\sigma)^{3 / 2}-f(1-\sigma)} \log t
\end{aligned}
$$

on defining $f(x)=18 x^{3 / 2}-(x-\varrho)(k-1)^{-1}, x>0$.

Simple calculations show that $f$ has a global minimum on $(0, \infty)$ at the point $x_{0}=(27(k-1))^{-2}$ and $f\left(x_{0}\right)=-\left(3^{7}(k-1)^{3}\right)^{-1}+\varrho(k-1)^{-1}$. 
It follows that

$$
\sum_{t^{1 / k}<n \leq t^{1 /(k-1)}}(n+\alpha)^{-s} \ll t^{18(1-\sigma)^{3 / 2}-f\left(x_{0}\right)} \log t .
$$

However, for $11 \leq k \leq 1000$ we can find that

$$
\begin{aligned}
f\left(x_{0}\right) & =-\left(3^{7}(k-1)^{3}\right)^{-1}+\left(3 k^{2}(k-1) \log 120 k\right)^{-1} \\
& =\frac{1}{3(k-1)^{3}}\left\{\left(\frac{k-1}{k}\right)^{2} \frac{1}{\log 120 k}-\frac{1}{3^{6}}\right\} \\
& \geq \frac{1}{3(k-1)^{3}}\left\{\left(\frac{10}{11}\right)^{2} \frac{1}{\log 120 \cdot 10^{3}}-\frac{1}{3^{6}}\right\}=\frac{1}{3(k-1)^{3}} \lambda_{1} .
\end{aligned}
$$

Numerical calculations show that $\lambda_{1}>0$ whence

(4)

$$
\sum_{t^{1 / k}<n \leq t^{1 /(k-1)}}(n+\alpha)^{-s} \ll t^{18(1-\sigma)^{3 / 2}} \log t \ll t^{18 \cdot 1(1-\sigma)^{3 / 2}} \log ^{2 / 3} t .
$$

From (1) and (2)-(4) we can easily conclude that

$\sum_{t^{1 / 1000}<n \leq t^{1 / 10}}(n+\alpha)^{-s}=\sum_{k=11}^{1000} \sum_{t^{1 / k}<n \leq t^{1 /(k-1)}}(n+\alpha)^{-s} \ll t^{18.1(1-\sigma)^{3 / 2}} \log ^{2 / 3} t$ for $0<\sigma<1-2^{-1002}$ and sufficiently large $t>0$.

Lemma 5 ([1], Lemma 6 , p. 15). Let $\sigma \geq 0, t \geq t_{8}>0, k, K, M, N \in \mathbb{N}$, $k \geq 2, K=2^{k}, N \leq M \leq 2 N$ and $1 \leq N \leq t^{2 / 3}$. Then

$$
\sum_{N \leq n \leq M}(n+\alpha)^{-s} \ll N^{1-\sigma-k /(K-2)} t^{1 /(K-2)} .
$$

Corollary 4. For $0<\sigma<1<2^{-1002}, t \geq t_{9}>0$ and $4 \leq k \leq 17$,

$$
\sum_{t^{1 /(k-1)}<n \leq t^{1 / 2}}(n+\alpha)^{-s} \ll t^{18 \cdot 1(1-\sigma)^{3 / 2}} \log ^{2 / 3} t .
$$

Pr o of. Taking $k \geq 4, Q=\left[t^{1 /(k-1)}\right]+1$, and defining $r$ as the largest integer such that $Q \cdot 2^{r} \leq t^{1 / 2}$ we have

$$
\begin{aligned}
\sum_{t^{1 /(k-1)<n \leq t^{1 / 2}}}(n+\alpha)^{-s} & \sum_{m=0}^{r-1} \sum_{Q \cdot 2^{m} \leq n \leq Q \cdot 2^{m+1}}(n+\alpha)^{-s}+\sum_{Q \cdot 2^{r} \leq n \leq t^{1 / 2}}(n+\alpha)^{-s} .
\end{aligned}
$$

From Lemma 5 we get

$$
\sum_{t^{1 /(k-1)}<n \leq t^{1 / 2}}(n+\alpha)^{-s} \ll \sum_{m=0}^{r}\left(Q \cdot 2^{m}\right)^{1-\sigma-k /(K-2)} t^{1 /(K-2)} .
$$


Clearly

$$
2^{-1002}-\frac{k}{K-2}<1-\sigma-\frac{k}{K-2}<1-\frac{k}{K-2}, \quad r \ll \log t .
$$

If $1-\sigma-k /(K-2) \leq 0$, then

$\sum_{t^{1 /(k-1)}<n \leq t^{1 / 2}}(n+\alpha)^{-s} \leq t^{\frac{1}{k-1}\left(1-\sigma-\frac{k}{K-2}\right)+\frac{1}{K-2}} \log t=t^{18(1-\sigma)^{3 / 2}-g(1-\sigma)} \log t$ on defining

$$
g(x)=18 x^{3 / 2}-\frac{x}{k-1}+\frac{1}{(K-2)(k-1)}, \quad x>0 .
$$

Just like for the function $f$ considered in the proof of Corollary 3 we find that $g$ has a global minimum on $(0, \infty)$ at $x_{0}=(27(k-1))^{-2}$ but $g\left(x_{0}\right)=-\left(3^{7}(k-1)^{3}\right)^{-1}+((K-2)(k-1))^{-1}$. It follows that

$$
\sum_{t^{1 /(k-1)}<n \leq t^{1 / 2}}(n+\alpha)^{-s} \ll t^{18(1-\sigma)^{3 / 2}-g\left(x_{0}\right)} \log t .
$$

However, for $4 \leq k \leq 19$, we can find that

$$
\begin{aligned}
g\left(x_{0}\right) & =-\left(3^{7}(k-1)^{3}\right)^{-1}+\left(\left(2^{k}-2\right)(k-1)\right)^{-1} \\
& =\frac{1}{(k-1)^{3}}\left\{\frac{(k-1)^{2}}{2^{k}-2}-\frac{1}{3^{7}}\right\} \\
& \geq \frac{1}{(k-1)^{3}}\left\{\frac{18^{2}}{2^{19}-2}-\frac{1}{3^{7}}\right\}=\frac{1}{(k-1)^{3}} \lambda_{2},
\end{aligned}
$$

and $\lambda_{2}>0$.

This yields

$$
\sum_{t^{1 /(k-1)}<n \leq t^{1 / 2}}(n+\alpha)^{-s} \ll t^{18(1-\sigma)^{3 / 2}} \log t \ll t^{18 \cdot 1(1-\sigma)^{3 / 2}} \log ^{2 / 3} t .
$$

Otherwise $(0<1-\sigma-k /(K-2))$

$\sum_{t^{1 /(k-1)}<n \leq t^{1 / 2}}(n+\alpha)^{-s} \ll t^{\frac{1}{2}\left(1-\sigma-\frac{k}{K-2}\right)+\frac{1}{K-2}} \log t=t^{18(1-\sigma)^{3 / 2}-h(1-\sigma)} \log t$ on defining $h(x)=18 x^{3 / 2}-x / 2+(k-2) /(2(K-2)), x>0$.

We find that the function $h$ has a global minimum on $(0, \infty)$ at the point $x_{0}=54^{-2}$ and $h\left(x_{0}\right)=-\left(2^{3} \cdot 3^{7}\right)^{-1}+(k-2) /(2(K-2))$.

A not very difficult calculation shows that for $4 \leq k \leq 17$,

$$
h\left(x_{0}\right)=-\frac{1}{2^{3} \cdot 3^{7}}+\frac{k-2}{2\left(2^{k}-2\right)} \geq \frac{15}{2\left(2^{17}-2\right)}-\frac{1}{2^{3} \cdot 3^{7}}>0 .
$$


It follows that

$$
\begin{aligned}
\sum_{t^{1 /(k-1)}<n \leq t^{1 / 2}}(n+\alpha)^{-s} & \ll t^{18(1-\sigma)^{3 / 2}-h\left(x_{0}\right)} \log t<t^{18(1-\sigma)^{3 / 2}} \log t \\
& \ll t^{18.1(1-\sigma)^{3 / 2}} \log ^{2 / 3} t .
\end{aligned}
$$

III. Proof of the Theorem. According to van der Corput and Koksma's result ([1], p. 4) we have

$$
\zeta(s, \alpha)-\alpha^{-s} \ll t^{\frac{1-\sigma}{\log (1 /(1-\sigma))} \log 2} \frac{\log t}{\log \log t}
$$

for $1 / 2 \leq \sigma<1$ and $t>e$.

This estimate is better than ours for $1 / 2 \leq \sigma \leq 1-1 / 2^{16}$ and sufficiently large $t$. Indeed, for $1 / 2 \leq \sigma \leq 1-1 / 2^{16}$ we have $1 / 2^{16} \leq 1-\sigma \leq 1 / 2$ and

$$
(1-\sigma) \log \frac{1}{1-\sigma} \log 2 \leq 16(1-\sigma)^{3 / 2} .
$$

It follows that for sufficiently large $t>t_{10}>0$,

$$
\zeta(s, \alpha)-\alpha^{-s} \ll t^{16(1-\sigma)^{3 / 2}} \frac{\log t}{\log \log t} \ll t^{16 \cdot 1(1-\sigma)^{3 / 2}} \log ^{2 / 3} t .
$$

Clearly it suffices to consider the range $1-1 / 2^{16}<\sigma \leq 1$.

For $1-2^{-5} \leq \sigma \leq 1$ and $t \geq t_{11}>0$ (compare [9], p. 101, and [8], pp. 270-271) we have

$$
\left|\zeta(s, \alpha)-\alpha^{-s}\right| \leq\left|\sum_{n \leq t^{1 / 2}}(n+\alpha)^{-s}\right|+\text { const }
$$

and

$$
\sum_{n \leq t^{1 / 2}}(n+\alpha)^{-s}=\left\{\sum_{n \leq N_{1}}+\sum_{N_{1}<n \leq N_{2}}+\sum_{N_{2}<n \leq N_{3}}+\sum_{N_{3}<n \leq t^{1 / 2}}\right\}(n+\alpha)^{-s}
$$

where $N_{1}=\exp \left(\log ^{2 / 3} t\right), N_{2}=t^{1 / 1000}, N_{3}=t^{1 / 10}$.

For the first sum we use Lemma 1. Corollary 1 gives an estimate for the second sum. To estimate the third and fourth term one can use Corollary 3 , Corollary 4 with $k=11$ and Corollary 2 . In this way our Theorem is proved.

Acknowledgements. I would like to thank Professor D. R. HeathBrown for some "instructions" concerning the scheme of the proof [3]. I would also like to thank Acta Arithmetica's referee for valuable remarks which enabled me to improve the final version of the paper. Finally I would like to thank Professor K. M. Bartz for suggesting this problem and for her help and assistance in preparing this paper. 


\section{References}

[1] J. G. van der Corput et J. F. Koksma, Sur l'ordre de grandeur de la fonction $\zeta(s)$ de Riemann dans la bande critique, Ann. Fac. Sci. Univ. Toulouse (3) 22 (1930), $1-39$.

[2] W. J. Ellison et M. Mendès-France, Les nombres premiers, Hermann, Paris, 1975.

[3] D. R. Heath-Brown, private correspondence, 1992.

[4] A. Ivić, The Riemann Zeta Function, Wiley, 1985.

[5] M. Kulas, Some effective estimation in the theory of the Hurwitz-zeta function, Funct. Approx. Comment. Math. 23 (1994), 123-134.

[6] E. I. Panteleeva, On a problem of Dirichlet divisors in number fields, Mat. Zametki 44 (1988), 494-505 (in Russian).

[7] -, On mean values of some arithmetical functions, ibid. 55 (1994), no. 2, 109-117 (in Russian).

[8] K. Prachar, Primzahlverteilung, Springer, Berlin, 1957.

[9] H. E. Richert, Zur Abschätzung der Riemannschen Zetafunktion in der Nähe der Vertikalen $\sigma=1$, Math. Ann. 169 (1967), 97-101.

[10] W. Staś, Über das Verhalten der Riemannschen $\zeta$-Funktion und einiger verwandter Funktionen, in der Nähe der Geraden $\sigma=1$, Acta Arith. 7 (1962), 217-224.

[11] E. C. Titchmarsh, The Theory of the Riemann Zeta-Function, Clarendon Press, Oxford, 1986.

[12] P. Turán, On some recent results in the analytical theory of numbers, in: Proc. Sympos. Pure Math. 20, Amer. Math. Soc., 1971, 339-347.

[13] O. V. Tyrina, A new estimate for a trigonometric integral of I. M. Vinogradov, Izv. Akad. Nauk SSSR Ser. Mat. 51 (1987), 363-378 (in Russian).

[14] I. M. Vinogradov, General theorems on the upper bound of the modulus of a trigonometric sum, ibid. 15 (1951), 109-130 (in Russian).

Faculty of Mathematics and Computer Science

Adam Mickiewicz University

Matejki 48/49, 60-769 Poznań, Poland

E-mail: kulas@math.amu.edu.pl 